\title{
PRESERVATION OF AUDITORY-EVOKED BRAINSTEM RESPONSES IN ANAESTHETIZED CHILDREN
}

\author{
P.G. Duncan, R.A. Sanders and D.W. McCullough
}

The usefulness of standard electroencephalogram monitoring in anaesthesia and critical care has been limited due to the influence of concommitantly administered drugs. It has been shown that a given anaesthetic drug will, under controlled conditions, produce a predictable. dose-related series of changes in scalp electrodes'. However, the clinical usefulness of this phenomenon is compromised since it has also been shown that the electroencephalogram pattern does not necessarily change in the same sequence with all anaesthetic drugs, that the same signal does not necessarily represent the same clinical state when achieved with different drugs, and that other physiological variables such as blood gas tension will modify the results. For these reasons many different modifications of the standard electroencephalogram have been advanced to ease its deployment as a clinical tool in various situations?

One such modification of the standard electroencephalogram is the sensory-evoked response where a recurrent signal is generated in response to repeated stimuli and extracted from the ongoing electroencephalogram. This technique has been used with tactile, visual, and auditory input. When used during states of anaesthesia there is generally depression of the cortical projection of the sensory stimulation with very low concentrations of anaesthetics and hypnotics.' The early (or specific) response to a given stimulus, with a neuronal origin more peripheral than the cortex, appears to be more resistant to alterations of the state of the subject and drugs administered, but is difficult to record by standard electroencephalographic techniques.

Evoked response audiometry represents a further refinement of the evoked electroencephalogram potential. ${ }^{3}$ It is based on the principle of computerized far-field averaging, where the generators of the electroencephalogram po-

P.G. Duncan, M.D., F.R.C.P.(C), Assistant Professor of Anaesthesia and Paediatrics. Department of Anaesthesia; R.A. Sanders. M.A.. Clinical Audiologist. Department of Otolaryngology; D.W McCullough, M.B., F.R.C.S.(C). Professor and Head. Department of Otolaryngology. University of Manitoba. Winnipeg. Manitoba. tentials (the cell bodies and dendrites of the central nervous system) are at a distance from recording electrodes on the scalp. The brain, being a more or less homogeneous medium, acts as a "volume conductor" of all the electrical impulses occurring inside the cranium and allows their recording by vertex electrodes. The far-field recording of the basal activity of the brain will demonstrate large ( $\mu$ volts) potentials with a frequency dependent on the state of the subject but unsynchronized in time. Such bipolar random dendretic potentials, when summated and averaged by computer, will approximate zero potential over time. Hence the averaged background activity of the central nervous system can be removed from the electroencephalogram. The auditory-evoked potential, on the other hand. is generated in response to recurring stimulation of the conducting pathway and, athough small (nanovolts) has a definite polarity and temporal relationship to the stimulus. Electrical enhancement of the recordings is achieved by appropriate artifact rejection circuits, electrical filtering, and acoustic and electrical masking. Thus, the evoked potential can be recognized and summated over multiple stimulations. By employing the appropriate time interval after stimulation for recording the electroencephalogram, the activity in designated anatomical areas of the auditory pathway can be examined.

We have been interested in using the electrical activity arising from the brainstem nuclei of the auditory pathway for objective assessment of hearing acuity, as well as for non-invasive monitoring of brainstem integrity. Since general anaesthesia had not been previously employed for brainstem evoked response audiometry, we sought to examine the feasibility of performing evoked response audiometry under anaesthesia and the influence of a standard general anaesthetic on the brainstem auditory-evoked response.

\section{METHODS}

Prior to the study, 30 awake volunteers with normal hearing were used to standardize our equipment and to establish our range of normality 92

Canad. Anaesth. Soc. J., vol. 26, no. 6. November 1979 


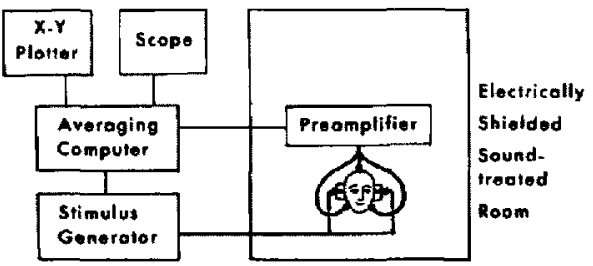

Figure I Schematic representation of the technique of evoked response audiometry. The defined stimulus is generated and administered to both ears by way of standard earphones. The electroencephalogram is recorded by three cutaneous electrodes, amplified, and the background activity removed by the averaging computer. The oscilloscope affords a constant monitor of the generated response, while the $X-Y$ recorder forms a permanent record after a run of stimulations. The need for acoustic and electrical isolation is repres. ented by the electrically shielded sound-treated room.

Six children two to nine years of age were referred for hearing assessment by evoked audiometry. All were part of a larger series of children who could not have their hearing adequately assessed by standard audiological techniques due to lack of co-operation or because of mental or physical disabilities. ${ }^{6}$ None of the chitdren had active neurological disease, and none of the six subjects were taking any medications. All were ASA class I anaesthetic risks.

The patients were studied as outpatients after an eight-hour fast. With no premedication, they were induced with 50 per cent nitrous oxide and halothane; their tracheae were intubated without relaxants, and anaesthesia was maintained with halothane in oxygen through a Jackson-Rees T-piece used as a non-rebreathing circuit. Temperature, hydration, oxygenation and end-tidal carbon dioxide were maintained normal throughout the period of assessment.

Evoked response audiometry was done as illustrated in Figure 1. A commercial evoked response audiometry instrument (Madsen ERA-74) delivered unfiltered square wave clicks of $0.1 \mathrm{msec}$ duration at $22 / \mathrm{sec}$ and an intensity of 60 decibels to both ears. Electroencephalogram activity was recorded from three scalp electrodes for $10 \mathrm{msec}$. beginning $1 \mathrm{msec}$ after the onset of each click. Computer-averaged responses were monitored on an oscilloscope for 1000 to 3000 clicks, after which the averaged activity was printed out on an $X-Y$ recorder. Presence of wave $V$ of the classical brainstem waveform was used to indicate an intact peripheral auditory system and brainstem pathway.

After demonstration of normal hearing (normal wave $V$ latency and amplitude) and es tablishment of normal auditory threshold, the effect of varied anaesthetic depth on the evoked response was determined. The subjects were exposed to 2.0 per cent inspired halothane in oxygen for 60 minutes as delivered by a calibrated vaporizer to the non-breathing system. Gas chromatography of end-tidal samples obtained by Rahn sampler confirmed the attainment of 85 per cent of inspired concentration by this time. After measurement of the brain stem response at this depth, the patients were serially equilibrated for $20 \mathrm{~min}$ utes with progressively decreasing halothane concentrations $(1.5,1.0,0.50,0.25$ per cent $)$, after which the brainstem response was repeated After assessment of halothane effect. thiopentone $4 \mathrm{mg} \cdot \mathrm{kg}^{-1}$ was administered intravenously and responses were measured immediately before, and one and five minutes after the drug.

Data on the latency of wave $V$ of the brainstem response were compared to our established norms as well as to values for each individual at other anaesthetic concentrations by Students t-test for unpaired data. Wave $V$ amplitude values were similarly compared to the internal $0.1 \mu \mathrm{V}$ standard of the computer.

\section{RESULTS}

The normal value for wave $V$ latency in our awake volunteers to a $60 \mathrm{~dB}$ unfiltered click was $5.7 \mathrm{msec}$ with a standard deviation of $0.26 \mathrm{msec}$. Latency data for wave $V$ at other intensities was also obtained and was found to be similar to published reports. ${ }^{3}$

All six patients in this series had normal hearing as assessed by brainstem-evoked responses. The one subject at the extreme upper edge of latency data had a concomitant serous otitis media and mild conductive hearing loss. At higher intensities his values were within the limits of normal. However, all the values shown are at a standard $60 \mathrm{~dB}$ intensity stimulus.

There was no variation in the brainstem response with change in anaesthetic depth over the range tested in this study. The latency of wave $V$ was normal over the range of equilibrated halothane concentrations (Figure 2), as was the amplitude when compared to the internal standard (Figure 3). Similar results were obtained after the thiopentone injection in all cases (Figure 4). Neither halothane or thiopentone in the concentrations tested was capable of causing deviation of the brainstem response from the established normal. 


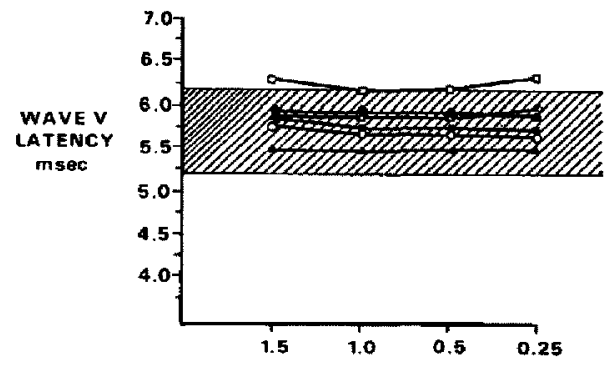

INSPIAED (HALOTHANE), VOL, $*$

Figure 2 Latency of wave $V$ at various equilibrated halothane concentrations for the six study subjects. The normal value ( \pm 2 S.D.) for latency as established in awake volunteers is represented by the shaded area.

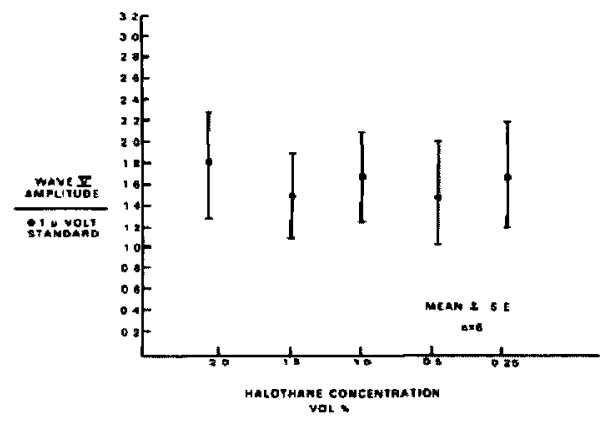

Figure 3 The ratio of waye $V$ amplitude to the internal computer standard at various halothane concentrations. There was no significant variation in amplitude ratio over the tested range of concentration.

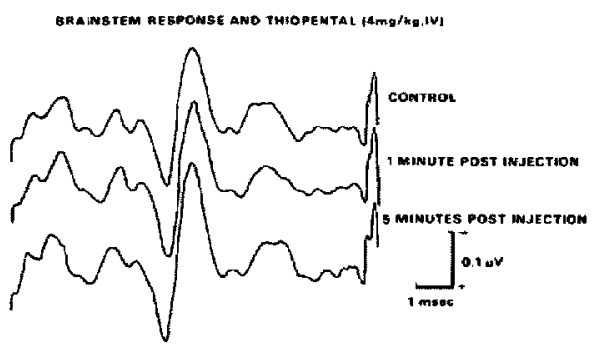

FIGUaE 4 Brainstem-evoked responses in a single individual before. one minute. and five minutes after intravenous thiopentone $4 \mathrm{mg} \cdot \mathrm{kg}^{-1}$. Wave $V$ is the largest peak of the tracing, and constant in amplitude and latency.

\section{Discussion}

The normal auditory-evoked response has a temporal pattern related to the origin of the electrical potential in the auditory pathway. The early (or fast) response (1-10 msec post-stimulation) has been established by ablation experiments in animals and confirmed by human studies ${ }^{5.6}$ to originate in the nuclei of the VIIl nerve and the auditory pathway. The middle response (12$-50 \mathrm{msec}$ ) includes the last brainstem response and first cortical projection of the stimulus. The cortical response $(50-250 \mathrm{msec})$ represents the secondary and tertiary cortical projection of the sound stimulus. Thus, by the use of the appropriate electrical timing filter, the technique of evoked response audiometry allows one to examine neuronal activity in any part of the conducting pathway from the VIII nerve to the auditory cortex.

We have elected in this report to evaluate the effects of anaesthesia on the early. or brainstem. response and used as our reference the conduction of auditory-induced electrical activity up to the level of the inferior colliculus (wave V). Our failure to demonstrate any effect of anaesthesia up to this level suggests a relative resistance of midbrain nuclei to the pharmacological effects of halothane or thiopentone. Similar data have been reported with althesin sedation in man' and halothane and methoxyflurane in cats, ${ }^{10}$ but other anaesthetic drugs have not been evaluated in a controlled fashion in man. In contrast to the resistance of the early response to anaesthetic effect, the late or cortical response is ablated by very small concentrations of sedatives and hypnotics. ${ }^{1.9}$ The middle response, the late brainstem or early cortical projection of acoustic stimulation, has not been thoroughly evaluated but has been shown to demonstrate a prolonged latency as a result of secobarbitone sedation ${ }^{10}$ or nitrous oxide/halothane anaesthesia."

The use of evoked response audiometry in the evaluation of hearing disorders is now well established, particularly in children. ${ }^{22}$ The latency of the responses varies inversely with age up to 18 months, after which time age is not a factor. The latency also varies inversely with the intensity of stimulation and, hence, standard test parameters must be employed. Although the influence of altered blood gas tensions on brainstem responses has not been examined. there is little reason to suspect an effect, since cortical projections of auditory stimulation have been shown to be intact 
over commonly observed ranges of carbon dioxide and oxygen. ${ }^{13}$ Our demonstration of the preservation of brainstem responses under halothane and thiopentone anaesthesia supports the feasibility of performing evoked response audiometry during full anaesthesia.

Since the preservation of normal audio-evoked responses at the brainstem level has been correlated with functional integrity of brainstem nuc$l e i^{14}$, there are several interesting speculative roles for this diagnostic modality in critical care. Since we have shown preservation of brainstem responses in spite of anaesthetizing concentrations of drugs, it is possible that evoked response audiometry may be useful to separate out the cause of depressed consciousness; an isopotential cortical electroencephalogram with preserved brainstem responses should suggest a toxic or pharmacological cause of coma ${ }^{15}$. Additionally, with the widespread utilization of paralysis, barbiturate "coma" and hyperventilation in the management of acute central nervous system insults, clinically reliable methods for assessment of brainstem function are lacking or obliterated by therapy. Evoked response audiometry may be a suitable noninvasive monitor of neuronal function during these situations. 16 However, such application remains speculative at present, to be explored by further clinical studies.

\section{Summary AND CONCLUSIONS}

In a series of six anaesthetized children we have been unable to demonstrate any effect of anaesthetic concentrations of halothane or thiopentone on the brainstem auditory-evoked response. The results imply preservation of brain stem function during anaesthesia with these drugs and suggest future application of anaesthesia to the testing of hearing disabilities in children, as well as application of evoked response audiometry to neurological critical care.

\section{RÉSUMÉ}

Chez six enfants anesthésiés, nous avons été incapables de démontrer un effet quelconque de concentrations anesthésiques d'halothane ou de thiopenthal sur la réponse du tronc cérébral à des stimuli auditifs. Ces résultats indiquent qu'il y a préservation de la fonction au niveau du tronc cérébral au cours d'anesthésie avec ces deux agents et laissent entrevoir une utilisation prochaine de l'anesthésie dans l'évaluation de problèmes auditifs chez l'enfant, ainsi qu'une application de ce même test au niveau des unités de soins intensifs neurologiques.

\section{REFERENCES}

I. Clark, D.L. \& Rosner, B.S. Neurophysiologic effects of general anesthetics. Anesthesiology 38 : 564-582 (1973).

2. McDowall, D.G. Monitoring the brain. Anes. thesiology 45: $117-134$ (1976).

3. Davis, H. Principles of electric response audiometry. Ann. Otol. 85 (Supp. 28) (1976).

4. The medical letter on drugs and therapeutics. $19(6)$ : 26-27 (1977).

5. Starr. A. \& Mamilton. A.E. Correlation between confirmed sites of neurological lesions and abnomalities of far-field auditory brainstem responses. Electroenceph. Clin. Neurophysiol. 4/; 595-608 (1976)

6. Stockard, J.J. \& Rossiter, V.S. Clinical and pathologic correlates of brain stem auditory response abnormalities. Neurology 27: 316-325 (1977).

7. Spillman, $T$ Erpmann, $W$ \& Leitner. $H$ Clinical experiences with althesin sedation for ERA. Revue de Laryngologie 96: 192-198 (1975).

8. Ketahats. L.M. The effects of halothane and methoxyflurane on recovery cycles of click-evoked potentials from the auditory cortex of the cat. Anesthesiology 29: 523-532 (1968).

9. Hosick. E.G. Mendel. M.J. Effects of secobarbital on the late components of the auditoryevaked potentials. Revue de Laryngologie 96: 185-190(1975)

10. Mendel. M.I. \& Hosick, E.C. Effects of secobarbital on the early components of the audituryevoked potentials. Revue de Laryngologie 96 : $178-183(1975)$.

11. Celesia, G. \& Puletri, F. Auditory input to the human cortex during states of drowsiness and surgical anesthesia. Electroenceph. Clin. Neurophysiol. 3I: 603-609 (1971).

12. Mokotoff, B., Schulman-Galambos, C. \& GALAMBOS, R. Brain stem auditory-evoked responses in children. Arch Otolaryngol. 103: 38-43 (1977).

13. Deecke, L., Goode, R. C. \& Whitehead, G. Effect of altered respiration on auditory-evoked potential in man. Prog. Clin. Neurophysiol. 2: 119-129 (1977)

14. StaRr, A. Auditory brain-stem responses in brain death. Hrain 99: 543-554 (1976).

15. StaRr. A. \& ACHOR, L.J. Auditory brain-stem responses in neurological disease. Arch Neurol. 32 $761-768$ (1975).

16. LARSEN, S.J. SANCES A. ACKMANN I.J. \& REIGEL, D.H. Noninvasive evaluation of head trauma patients. Surgery 72: 34-40 (1973). 